\title{
Energy Efficient Hybrid MAC Protocol for Cluster-Based Wireless Sensor Network
}

\author{
J. Vidhya, G. Kalpana and P. Dananjayan*
}

\begin{abstract}
Wireless sensor networks consist of large number of nodes often battery operated, which have ad hoc topology and variable traffic rates. The medium access control (MAC) protocol designed for the network must be stable and should consume less energy. This paper presents a novel hybrid MAC protocol that uses the information of clusters to divide the MAC layer functionality into two types, namely, bit map assisted (BMA) protocol and nanoMAC protocol for the intra and inter-cluster communication respectively. Performance of the hybrid MAC protocol is evaluated in terms of energy, delay and offered traffic load and compared with the conventional MAC schemes. Simulation results show that the hybrid MAC protocol considerably reduces the energy consumption and delay to maximize the lifetime of the sensor network.
\end{abstract}

Key words - BMA, energy efficiency, nanoMAC, wireless sensor networks.

\section{INTRODUCTION}

wireless sensor networks (WSN) have been envisioned to have a wide range of applications such as military, environmental monitoring and other fields [1]. Sensor nodes are characterized by their limited memory, power resource and computational capabilities. The traffic in WSN is often bursty with long inactivity periods. As a consequence, the device may remain idle for long periods of time, thus affecting the design of effective medium access protocols.

Medium access control schemes for wireless data communication networks are classified into two categories i.e., contention-based and schedule based protocols [2]. In contention-based scheme, the sensor nodes keep their radios on to transmit the message by sensing the channel. In mechanism such as non-persistence carrier sense multiple access (np-CSMA) protocol, nodes compete to share the channel but suffers from collisions resulting from nodes beyond one hop and leads to the hidden terminal problem [3]. Schedule based MAC scheme such as time division multiple access (TDMA), solves hidden terminal problem but requires efficient time schedule for data transmission to avoid idle listening to the channel [4]. The key sources of energy wastage in np-CSMA and TDMA MAC schemes results from collisions, overhearing control packet overhead and idle listening to the wireless medium [3], [4]. Thus an efficient MAC protocol which consumes less energy and acceptable throughput has to be suggested for handling the dynamic traffic in sensor network.

A TDMA based MAC scheme i.e., BMA MAC protocol

*Corresponding author is with the Department of Electronics and Communication Engineering, Pondicherry Engineering College, Puducherry, India. is proposed for this high contention resolution. It incorporates dynamic scheduling of non-cluster head nodes and when utilized for intra-cluster communication [5], it can significantly reduce the idle listening of nodes and conserve energy. With low contention, TDMA based MAC protocols give much lower channel utilization, higher delay and energy consumption compared with CSMA [5], [6]. In inter-cluster communication, channel contention is less, hence a CSMA based MAC protocol called nanoMAC is proposed. NanoMAC protocol incorporates sophisticated sleep algorithm and collision avoidance techniques with CSMA to minimize energy consumption. Hence this paper suggests a novel hybrid MAC approach with BMA and nanoMAC and utilizes clustering scheme to reduce energy consumption. The main feature of the hybrid MAC protocol is that it can adapt to the level of contention in the network either high or low. The analysis of the proposed hybrid MAC protocol is evaluated to maximize the lifetime of the network and compared with np-CSMA and conventional TDMA schemes.

The reminder of the paper is organized as follows. Section II describes the system model. Section III presents the hybrid MAC protocol scheme and its energy model for the intra and inter-cluster domain. The simulation framework and results are discussed in section IV and finally section $\mathrm{V}$ presents the conclusion of the suggested method.

\section{SYSTEM MODEL}

Clustering scheme organizes the nodes of the sensor network into two virtual domains, such as intra-cluster and inter-cluster domain and is shown in Fig.1. In the intra-cluster domain, the nodes within the cluster i.e. non-cluster head nodes sense the data and communicates with the cluster head directly [5]. There are large numbers of sensor nodes within the cluster which have higher probability of simultaneous transmissions to the cluster head. Since the radio channel has high contention in the intra-cluster domain, the TDMA based MAC (BMA) protocol is utilized for achieving high energy efficiency.

In the inter-cluster domain, the cluster head node communicates with the base station directly (single hop) or through other cluster head nodes (multi hop). However, its channel contention is less compared to intra-cluster. Hence CSMA based MAC (nanoMAC) protocol, the more appropriate solution for the inter-cluster domain is used. As each node in the cluster has equal chance of becoming as a cluster-head, all nodes in the network are equipped with both BMA and nanoMAC controllers [4], [6]. 


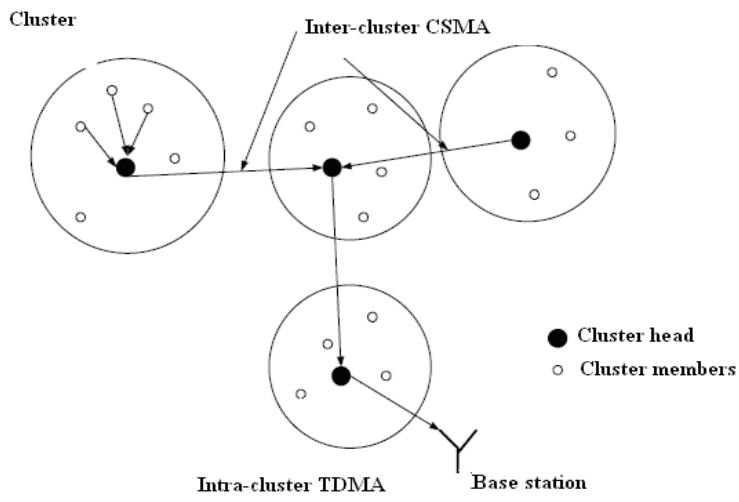

Fig.1. System model of hybrid MAC protocol

The frame structure of the hybrid MAC protocol is shown in Fig.2. The time slot is subdivided into mini-slots which carries one-bit information of each node to determine whether they have the sensed data or not. If the node has no sensed data its time slot is allocated to other nodes that have data and the cluster head assigns this schedule to its members, in the intra-cluster domain. Unlike intra-cluster, in inter-cluster domain nodes that have data to transmit performs carrier sense (CS) before transmission. If a node fails to get the medium it goes to sleep and wakes up after a random time period and listens for the channel again.

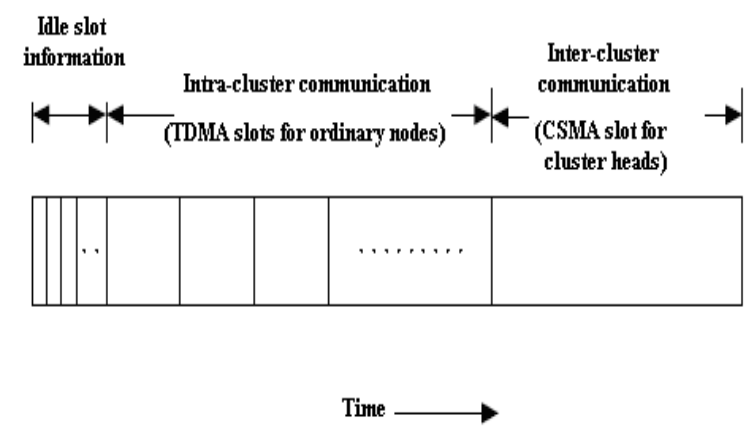

Fig.2. Hybrid MAC frame structure

The distinctive feature of the hybrid MAC protocol is its robustness to dynamic topology changes and time synchronization problems commonly occurring in the sensor network. The associated energy consumption and delay of the hybrid MAC protocol can be greatly minimized by the use of BMA MAC for intra-cluster and nanoMAC for inter-cluster domain.

\section{HYBRID MAC PROTOCOL}

\section{A. BMA MAC protocol for intra-cluster domain}

In conventional TDMA scheme, a node turns on its radio during its assigned slot regardless whether it has data to transmit or not. This results in high energy consumption when utilized for sensor nodes. The energy efficient TDMA (E-TDMA) scheme extends the conventional TDMA approach, a node turns its radio off when it has no data to transmit during its allocated time slots. This greatly reduces the energy consumption of sensor nodes compared to TDMA. Changing time slot allocations and frame lengths according to unpredictable variations of traffic in sensor network is usually hard using TDMA and E-TDMA.
Considering these constraints, the robust intra-cluster MAC protocol, BMA is suggested to achieve high energy efficiency. In clustering approach, the data transmission of the non-cluster head nodes is organized into rounds [7]. Each round consists of cluster set-up phase and steady-state phase as shown in Fig.3. During set-up phase, each node decides whether to become as cluster head based on its energy level.

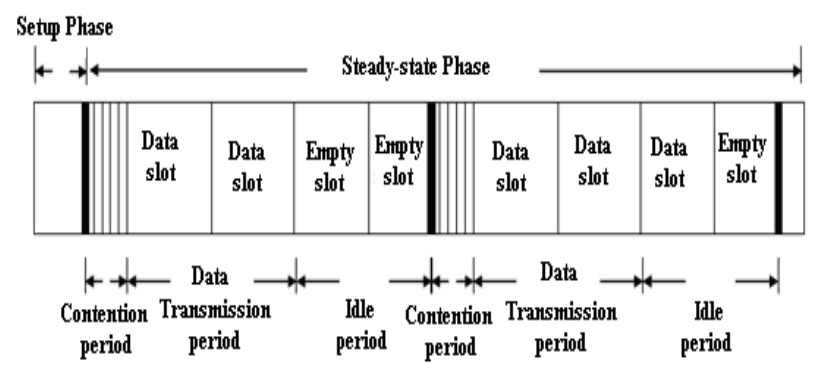

Fig.3. Transmission periods of BMA MAC protocol

The steady-state phase is divided into sessions. Each session consists of a contention period, a data transmission period and idle period. During the contention period, all nodes keep their radios on. Using BMA MAC each node is assigned a specific slot to transmit a 1-bit control message if it has data to send, otherwise, its scheduled slot remains empty. After the contention period, the cluster head broadcasts its transmission schedule to the non-cluster head nodes in the cluster and the system enters into the data transmission period. If none of the non-cluster head nodes have data to send, the system proceeds directly to an idle period, which lasts until the next session. The nodes keep their radios off during the idle periods to save energy.

The energy consumed by each source node during a single session is expressed as

$$
\mathrm{E}_{\mathrm{sn}}=\mathrm{P}_{\mathrm{t}} \mathrm{T}_{\mathrm{c}}+(\mathrm{N}-1) \mathrm{P}_{\mathrm{i}} \mathrm{T}_{\mathrm{c}}+\mathrm{P}_{\mathrm{r}} \mathrm{T}_{\mathrm{ch}}+\mathrm{P}_{\mathrm{t}} \mathrm{T}_{\mathrm{d}}
$$

where $\mathrm{N}$ is the number of non-cluster head nodes within a cluster, $\mathrm{P}_{t}, \mathrm{P}_{\mathrm{r}}$ and $\mathrm{P}_{\mathrm{i}}$ is the power consumption during the transmission, reception and idle mode, $\mathrm{T}_{\mathrm{c}}$ is the time required to transmit/receive a control packet, $T_{d}$ is the time required to transmit/receive a data packet and $\mathrm{T}_{\mathrm{ch}}$ is the time required for BMA cluster-head to transmit a control packet.

The energy dissipated during a single session by the non-source node is given by

$\mathrm{E}_{\mathrm{in}}=\mathrm{NP}_{\mathrm{i}} \mathrm{T}_{\mathrm{c}}+\mathrm{P}_{\mathrm{r}} \mathrm{T}_{\mathrm{ch}}$

During the contention period of the $i^{\text {th }}$ session, cluster head node receives $n_{i}$ control packets from non-cluster head nodes and stays idle for remaining contention slots. In the subsequent transmission period, the cluster head node receives $n_{i}$ data packets from the non-cluster head nodes. Hence, the energy expended in the cluster-head node during a single session is given as

$\mathrm{E}_{\mathrm{ch}}=\mathrm{n}_{\mathrm{i}}\left(\mathrm{P}_{\mathrm{r}} \mathrm{T}_{\mathrm{c}}+\mathrm{P}_{\mathrm{r}} \mathrm{T}_{\mathrm{d}}\right)+\left(\mathrm{N}-\mathrm{n}_{\mathrm{i}}\right) \mathrm{P}_{\mathrm{i}} \mathrm{T}_{\mathrm{c}}+\mathrm{P}_{\mathrm{t}} \mathrm{T}_{\mathrm{ch}}$ 
Therefore, the total energy consumed in each cluster during the $\mathrm{i}^{\text {th }}$ session is

$\mathrm{E}_{\mathrm{si}}=\mathrm{n}_{\mathrm{i}} \mathrm{E}_{\mathrm{sn}}+\left(\mathrm{N}-\mathrm{n}_{\mathrm{i}}\right) \mathrm{E}_{\mathrm{in}}+\mathrm{E}_{\mathrm{ch}}$

Each round consists of $\mathrm{k}$ sessions, thus the total system energy dissipated during each round is computed as

$\mathrm{E}_{\text {round }}=\sum_{\mathrm{i}=1}^{\mathrm{k}} \mathrm{E}_{\mathrm{si}}$

and hence, the average system energy expended during each round can be expressed as

$\mathrm{E}=\mathrm{E}\left[\mathrm{E}_{\text {round }}\right]=\mathrm{k}\left[\mathrm{n}_{\mathrm{i}} \mathrm{E}_{\mathrm{sn}}+\left(\mathrm{N}-\mathrm{n}_{\mathrm{i}}\right) \mathrm{E}_{\text {in }}+\mathrm{E}_{\mathrm{ch}}\right]$

The average packet delay $\mathrm{D}$, is defined as the average time required for a packet to be generated by a source node and received by the cluster-head node and is given by,

$\mathrm{D}=\frac{\mathrm{NT}_{\mathrm{c}}+\mathrm{T}_{\mathrm{ch}}+\mathrm{n}_{\mathrm{i}} \mathrm{T}_{\mathrm{d}}}{\mathrm{n}_{\mathrm{i}}}$

\section{B. NanoMAC protocol for inter-cluster domain}

In conventional np-CSMA scheme [3], a node with a frame to transmit senses the channel using carrier sense. If the channel is detected busy, the node waits for a random time interval for transmission to avoid collision. When two users sense the channel idle at same time and transmit their frames, collision occurs. This results in high energy consumption of the sensor node. To minimize the energy consumption, nanoMAC protocol is suggested as a feasible solution for inter-cluster domain.

NanoMAC protocol is of carrier sense nultiple access with collision avoidance (CSMA/CA) type and is non-persistent. With probability $\mathrm{p}$, the protocol will act as non-persistent and with probability 1-p, the protocol will refrain from sending even before $\mathrm{CS}$ and schedule a new time to attempt for CS [8], [9]. Nodes contending for the channel do not constantly listen for the channel, but sleep during the random contention window. When the backoff timer expires, the nodes wake up to sense the channel. This feature makes the CS time for nanoMAC short, and saves the energy of sensor nodes to a greater extent. With one request-to-send (RTS) and clear-to-send (CTS) reservation, a maximum of 10 data frames can be transmitted using the frame train structure as shown in Fig.4. The data frames are acknowledged by a single, common acknowledgement (ACK) frame that has a separate ACK bit reserved for each frame. In this way, only the corrupted frames are retransmitted and not the whole packet.

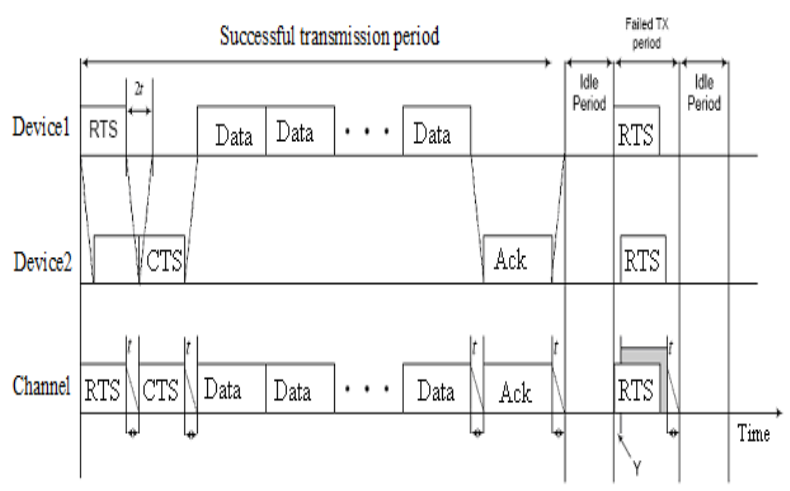

Fig.4. Transmission periods of nanoMAC protocol

The transmission energy consumption by the cluster head node for the inter-cluster domain with nanoMAC protocol is shown in Fig.5. This model describes the energy consumed during data transmission taking into account the average contention times, backoff times and frame collisions [10]. There are four different states: Arrive, Backoff, Attempt and Success state. Arrive state is an entry point to the system for a node to transmit new data. On every arrival to one of these states, energy is consumed. To reach the success state, all possible transitions starting from the arrival state and ending at the success state is calculated.

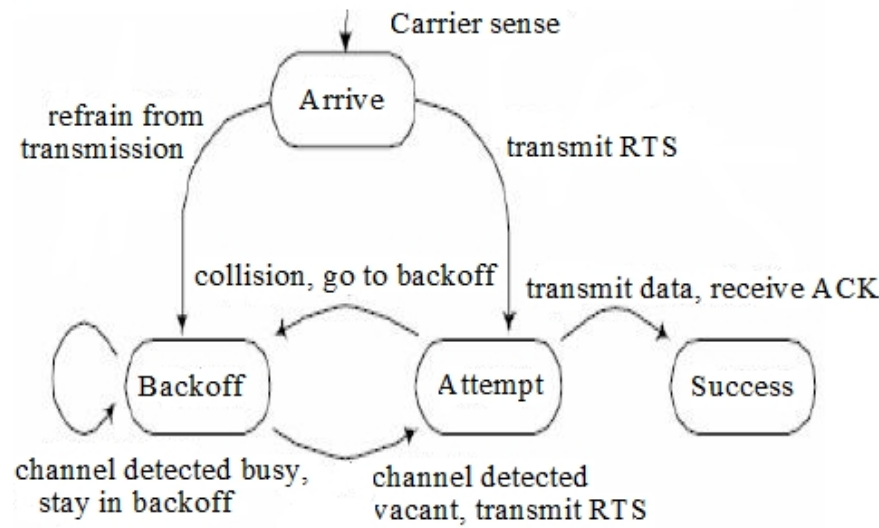

Fig.5. Transmitter energy model of nanoMAC protocol

On the arrival of data, when a device finds the channel busy, it refrains from its transmission, and reaches the backoff state. When the channel is clear upon CS, the sensor node transmits an RTS frame to the destination node and it waits for a CTS frame and reaches the attempt state. On successful transmission of the RTS and reception of CTS, a transition to the success state is made. The success state represents a successful data exchange with the destination When the RTS frame collides as shown in Figs.4 and 5, the device returns to the backoff state and no new data transmissions are made during this failed period.

Backoff state represents the device's waiting period, trying to acquire the channel again [10]. When the device detects the channel as vacant or idle, it transits to the attempt state by sending a RTS frame. When the channel is detected busy, it stays in the backoff state and the process repeats. Let $\mathrm{E}_{\mathrm{TX}}$ be the average transmitter energy consumption by a node with new data at the arrive state until the node reaches success state, the point of receiving an acknowledgement frame and is given by

$\mathrm{E}_{\mathrm{TX}}=\mathrm{E}_{\text {arrive }}+\mathrm{P}_{\text {prob } 1} \mathrm{E}(\mathrm{A})+\left(1-\mathrm{P}_{\text {prob1 }}\right) \mathrm{E}(\mathrm{B})$

where $\mathrm{E}_{\text {arrive }}$ is the carrier sensing energy consumption when reaching the arrive state, $\mathrm{E}(\mathrm{A})$ and $\mathrm{E}(\mathrm{B})$ are the energy consumption on each visit by the node to attempt state and backoff state and is given by

$\mathrm{E}(\mathrm{A})=\mathrm{P}_{\text {prob2 }} \mathrm{E}_{\text {success }}+\left(1-\mathrm{P}_{\text {prob2 }}\right) \mathrm{E}(\mathrm{B})$

and $\mathrm{E}(\mathrm{B})=\mathrm{P}_{\text {prob3 }} \mathrm{E}(\mathrm{A})+\left(1-\mathrm{P}_{\text {prob3 }}\right) \mathrm{E}(\mathrm{B})$ 
$\mathrm{E}_{\text {success }}$ is the expected energy consumption upon reaching the success state from the attempt state and $\mathrm{P}_{\operatorname{prob}\{1,2,3\}}$ are the different probabilities related to arriving to a certain state. The transmitter energy consumption can be simplified as

$$
\begin{aligned}
& \mathrm{E}_{\mathrm{TX}}=\mathrm{T}_{\mathrm{CS}} \mathrm{M}_{\mathrm{RX}}+\mathrm{P}_{\mathrm{b}}\left(\mathrm{T}_{\mathrm{bb}}+\frac{\mathrm{T}_{\mathrm{r}}}{2}\right) \mathrm{M}_{\mathrm{Slp}}+\mathrm{P}_{\mathrm{b}} \mathrm{E}(\mathrm{B})+ \\
& \left(1-\mathrm{P}_{\mathrm{b}}\right)\left(1-\mathrm{P}_{\mathrm{ers}}\right)\left(\mathrm{T}_{\mathrm{bp}}+\frac{\mathrm{T}_{\mathrm{r}}}{2}\right) \mathrm{M}_{\mathrm{Slp}} \\
& +\left(1-\mathrm{P}_{\mathrm{b}}\right) \mathrm{P}_{\mathrm{ers}} \mathrm{E}(\mathrm{A})+\left(1-\mathrm{P}_{\mathrm{b}}\right) \mathrm{P}_{\mathrm{ers}}\left(\mathrm{T}_{\mathrm{pr}}+\mathrm{RTS}\right) \mathrm{M}_{\mathrm{TX}} \\
& +\left(1-\mathrm{P}_{\mathrm{b}}\right)\left(1-\mathrm{P}_{\mathrm{ers}}\right) \mathrm{E}(\mathrm{B})
\end{aligned}
$$

where $T_{C S}$ is the time required for carrier sensing, $M_{R X}$ is the receiver power consumption, $\mathrm{P}_{\mathrm{b}}$ is the probability of finding channel busy during carrier sense, $\mathrm{T}_{\mathrm{bb}}$ is the incremented backoff time, $\mathrm{T}_{\mathrm{r}} / 2$ is the average random delay, $\mathrm{M}_{\mathrm{TX}}$ is the transmitter power consumption, $\mathrm{M}_{\mathrm{Slp}}$ is the sleep power consumption of transceiver, $\mathrm{T}_{\mathrm{pr}}$ is the time required to transmit a preamble, $P_{e r s}$ is the non-persistence value of nanoMAC, $\mathrm{T}_{\mathrm{bp}}$ is the un-incremented backoff time and RTS is the time required to transmit an RTS frame.

The receiver energy consumption of a packet for nanoMAC protocol is shown in Fig.6. This model takes the reception of data into account as the average probability of receiving the data correctly [10]. There are three different states: Idle, Reply and Received. When an RTS packet is received by the destination node, it transits to state Reply and forwards the CTS packet to the source. When the destination node receives the valid data packet from the source it reaches the received state and sends an ACK frame to the source node. When the CTS packet transmitted by the receiver collides it stays in Idle state.

The average receiver energy consumption $\mathrm{E}_{\mathrm{RX}}$ listening for a transmission to detect and receive a packet for being the proper destination is given by

$$
\mathrm{E}_{\mathrm{RX}}=\mathrm{E}(\mathrm{I})=\left(\mu+\mathrm{P}_{\mathrm{s}} \theta\right)\left(\mathrm{P}_{\mathrm{s}} \mathrm{P}_{\text {senh }}\right)^{-1}
$$

where $\mathrm{E}(\mathrm{I})$ is the energy incurred in each visit of node to idle state, $\mu$ represents the energy model transitions from state idle, $\theta$ represents the energy model transitions from state reply, $\mathrm{P}_{\mathrm{s}}$ and $\mathrm{P}_{\text {senh }}$ are the probabilities of no collision during RTS or CTS transmission.

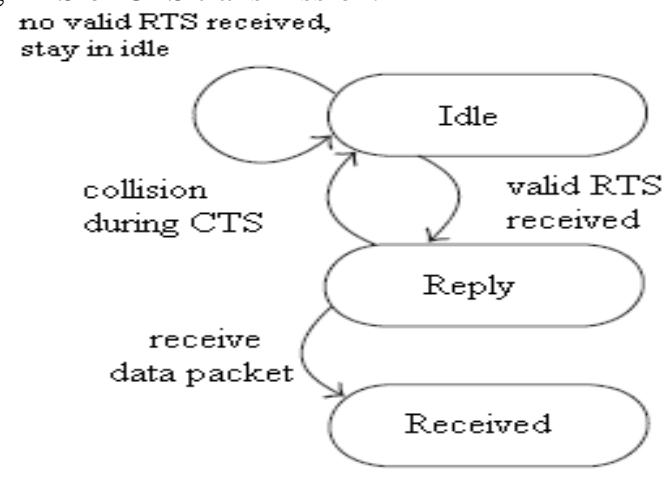

Fig.6 Receiver energy model of nanoMAC protocol

The average packet delay $\mathrm{D}$, from the cluster head to the base station is calculated using Fig. 5 and is given by

$$
\begin{aligned}
& \mathrm{D}=\mathrm{P}_{\mathrm{b}}\left[\mathrm{T}_{\mathrm{bb}}+\frac{\mathrm{T}_{\mathrm{r}}}{2}+\mathrm{E}(\mathrm{B})\right]+\left(1-\mathrm{P}_{\mathrm{b}}\right)\left(1-\mathrm{P}_{\mathrm{ers}}\right) \\
& {\left[\mathrm{T}_{\mathrm{bp}}+\frac{\mathrm{T}_{\mathrm{r}}}{2}+\mathrm{E}(\mathrm{B})\right]+\left(1-\mathrm{P}_{\mathrm{b}}\right) \mathrm{P}_{\mathrm{ers}}[\mathrm{E}(\mathrm{A})]}
\end{aligned}
$$

The channel throughput $\mathrm{S}$, is defined as the average number of successful frame transmissions per time interval $T_{p}$ and is given by

$$
S=\frac{G(b+1)\left(1-P_{e r s}+e^{-a G} P_{\text {ers }}\right)}{G\left(1+\left(4+P_{e r s}\right) a+2 b+c\right)+P_{e r s} e^{-a G}}
$$

where $\mathrm{G}$ is the traffic intensity or normalized traffic offered to the channel, $a$ is the normalized propagation time, $b$ is the normalized control packets and $\mathrm{c}$ is the normalized ACK delay.

\section{Simulation RESUlts}

The analysis of hybrid MAC protocol is carried out using MATLAB 7.1. The parameters considered for the simulation is summarized in Table.1. The performance of the intra-cluster BMA MAC protocol and the inter-cluster nanoMAC protocol are evaluated interms of traffic offered in the network, delay and energy consumption.

\begin{tabular}{|l|l|}
\multicolumn{1}{|c|}{ PABLE I SIMULATION PARAMETERS } \\
\begin{tabular}{|l|l|}
\hline Control frame size of nanoMAC & 18 bytes \\
\hline Data frame size of nanoMAC & 41 bytes \\
\hline Data frame payload of nanoMAC & 35 bytes \\
\hline Data packet size of BMA & 1400 bytes \\
\hline Data packet payload of BMA & 52 bytes \\
\hline Control frame size of BMA & 72 bytes \\
\hline Number of non-cluster head nodes & 20 to 45 nodes/cluster \\
\hline Device transmission range & $100 \mathrm{~m}$ \\
\hline
\end{tabular}
\end{tabular}

Fig.7. shows the average intra-cluster energy consumption with traffic load for a single round. A comparison is made for the three schedule based MAC schemes such as TDMA, E-TDMA and BMA protocol with 20 non-cluster head nodes in a cluster and four sessions/round. BMA is shown to provide better performance in terms of energy than E-TDMA and TDMA for traffic load less than 0.4. The energy consumption of BMA is 5\% less than E-TDMA and is $20 \%$ less than TDMA. The main reason for energy conservation in BMA protocol comes from avoiding idle listening for transmission through the channel. For traffic load greater than 0.4 , the idle period of BMA protocol is small and thus the energy cost from contention period outweighs the energy savings from idle periods. Thus for the traffic loads above 0.4, TDMA scheme performs better. The energy savings by E-TDMA and TDMA schemes relative of BMA grows as the traffic load approaches zero. 


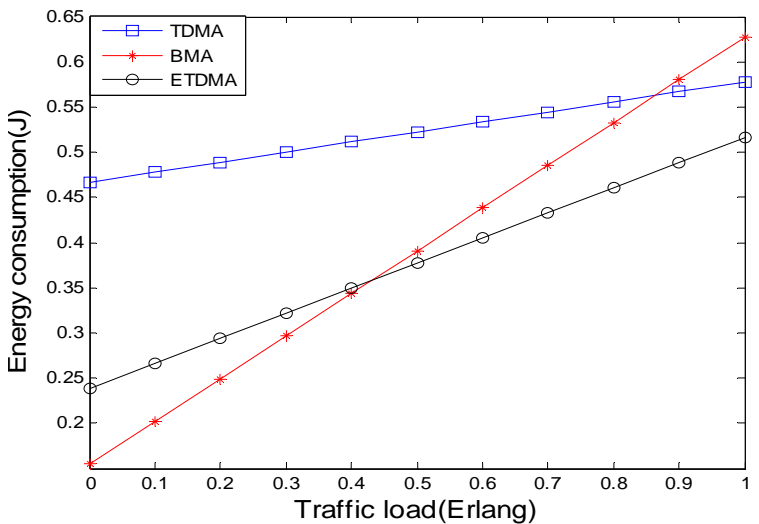

Fig.7. Comparison of intra-cluster energy consumed with traffic load

The performance of TDMA, E-TDMA and BMA protocols in terms of average intra-cluster energy expenditure with increasing number of sessions per round with 20 non-cluster head nodes in a cluster and for traffic load 0.3 is illustrated in Fig.8. It is vivid through the results that BMA has better performance when the number of sessions per round less than four. This is because the sensor nodes forward their data to the cluster head only if significant events occur. It is observed that using BMA protocol there is 5\% reduction in energy consumption compared to E-TDMA and 30\% reduction in energy consumption compared to TDMA scheme. When the number of sessions is greater than four, the energy consumption of BMA grows because of the increase in contention slots per round.

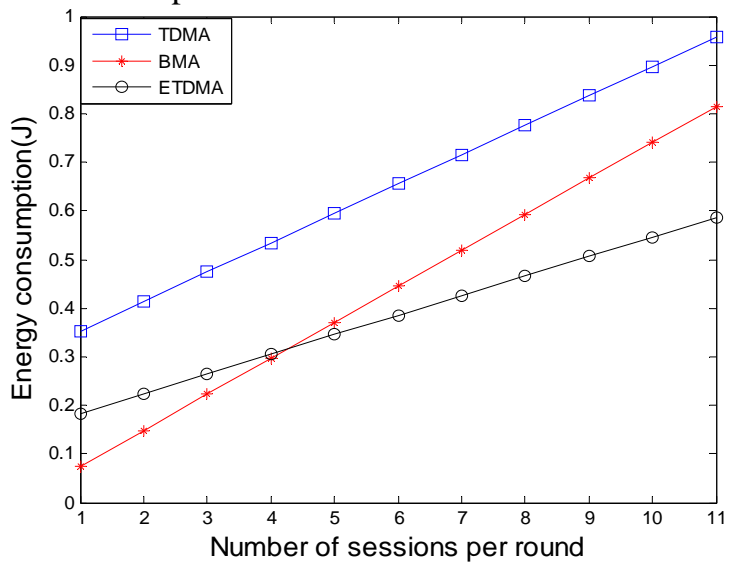

Fig.8. Comparison of intra-cluster energy consumption with sessions/round

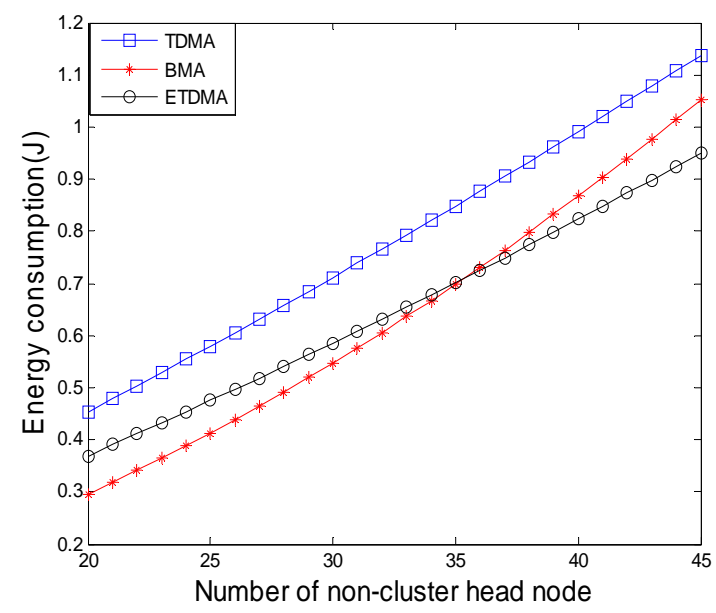

Fig.9. Intra-cluster energy consumption with non-cluster head nodes
The average intra-cluster energy consumption with the number of non-cluster head nodes in a cluster for traffic load 0.3 with four sessions per round is shown in Fig.9. When the number of non-cluster head nodes managed by the cluster head node is less than 35, BMA protocol performs better than E-TDMA and TDMA schemes. As the number of non-cluster head nodes in the cluster is larger, the contention period increases which results in greater energy consumption. Thus the optimum number of non-cluster head nodes for a cluster adapting BMA scheme is 35 .

Fig.10 compares the three MAC techniques in terms of average packet delay. For larger traffic load, all the three schemes provide less delay and are almost the same. However as the traffic load approaches minimum, the average packet delay grows exponentially with conventional TDMA and E-TDMA than BMA scheme. This is because in BMA protocol, the scheduling of nodes changes dynamically according to the traffic variations in the network. This greatly reduces the energy consumption of nodes due to idle listening and thus maintains a good and lower delay performance.

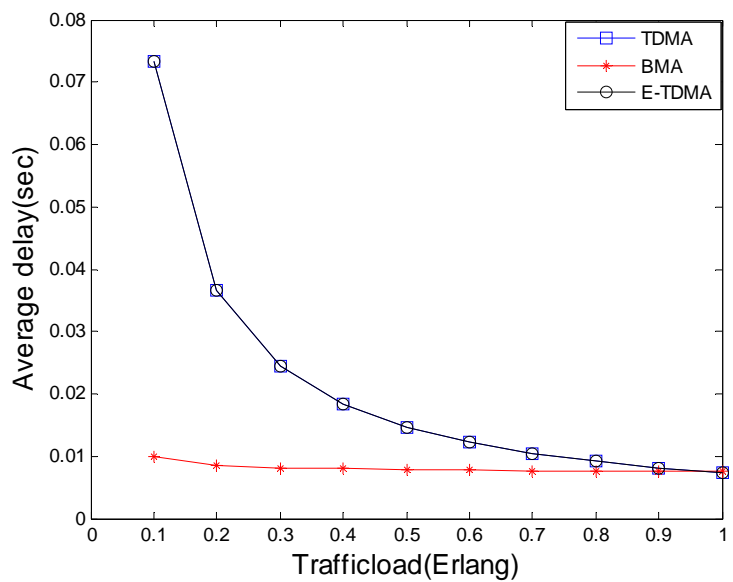

Fig.10. Intra-cluster average packet delay

The energy consumption in inter-cluster communication is due to transmission of packets by cluster head nodes to the base station. Fig.11 illustrates the energy consumption in transmission of data as a function of traffic load for 0.01 non- persistence nanoMAC and np-CSMA scheme. The transmission energy consumption of np-CSMA scheme is $40 \%$ more than nanoMAC. This is because np-CSMA protocol does not use frame train structure and nodes do not sleep during an over heard data transmission period and hence its energy consumption on transmission increases very rapidly. NanoMAC on the other hand performs well even in periods of high traffic bursts and its energy consumption stays low by incorporating proper sleep schedules. Thus nanoMAC protocol when used for inter-cluster domain can achieve better energy efficiency.

Fig.12 shows the throughput performance comparison of np-CSMA and 0.01 non-persistence nanoMAC protocol. When all the data frames are 410 bytes, nanoMAC protocol performs better as it sends ten data frames of 41 bytes each and an ACK frame instead of one 410 byte data frame and retransmits only the lost frame. This adds a multiplier of 10 to the throughput until the limit set by the data frame traffic is reached. It is vivid from the results that nanoMAC outperforms np-CSMA scheme in terms of throughput and 
makes the protocol more stable.

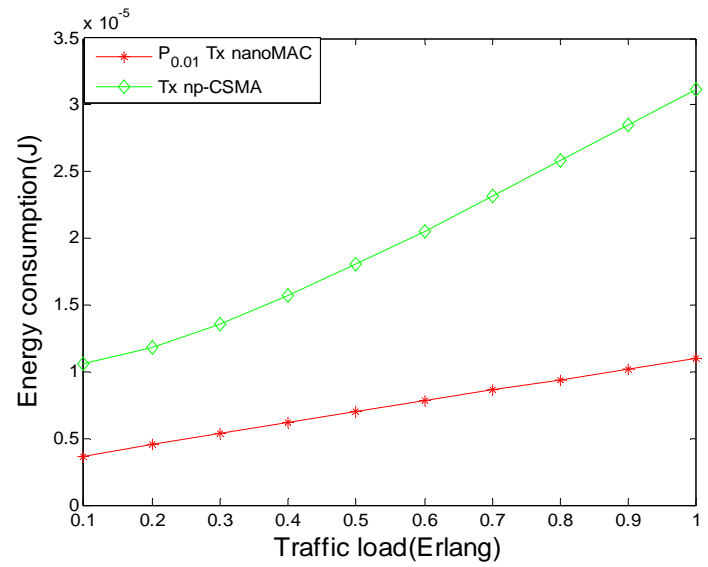

Fig.11. Inter-cluster energy consumption with traffic load

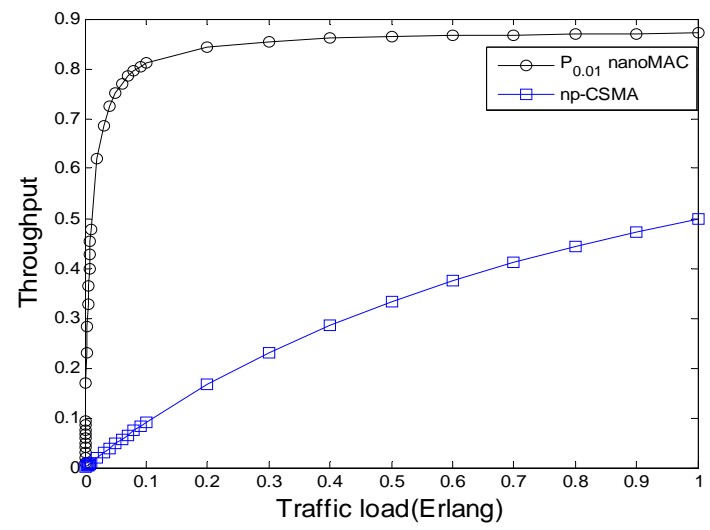

Fig.12. Comparison of throughput with traffic load for the inter-cluster

A comparison of normalized delay characteristics of 0.01 non-persistent nanoMAC and non-persistent CSMA protocols are shown in Fig.13. Using np-CSMA scheme, a device sends a single frame of 410 bytes and their corresponding acknowledgement frames in one transmission period. Upon error or collision during this transmission period, the entire frame has to be retransmitted, hence the delay incurred in reception of frame gradually increases with traffic load. With nanoMAC protocol, a device sends 10 data frames of 41 bytes each, an ACK frame for the same transmission period and retransmits only the lost/collided frame, thus the delay offered in the network is 15 times less compared to non-persistence CSMA. Furthermore, with nanoMAC protocol, when the probability of non-persistence decreases the sensor node has greater possibility to sense the channel and transmit the frame, thus its delay incurred in transmission of data decreases considerably.

Sensor nodes make use of single hop communication between cluster heads and base station. When the distance to reach the destination is increased, multihop communication between the cluster heads and base station is essential to minimize energy consumption [11]. Fig.14 shows the energy analysis of np-CSMA and nanoMAC protocol using single hop and multi hop communication between cluster head nodes. From the graph it is evident that single hop using nanoMAC protocol is best up to a transmission distance of $100 \mathrm{~m}$. As the distance increases above single hop, the energy consumption is increased approximately by a factor of 0.5. Mutihop using nanoMAC is more attractive and energy efficient when the transmission distance is beyond $100 \mathrm{~m}$ (10 hops). Thus nanoMAC protocol can achieve better efficiency when compared to np-CSMA.

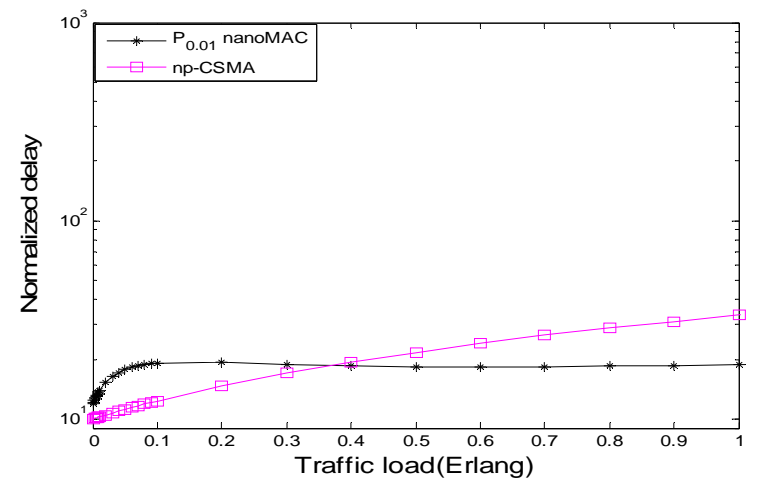

Fig.13. Inter-cluster packet delay

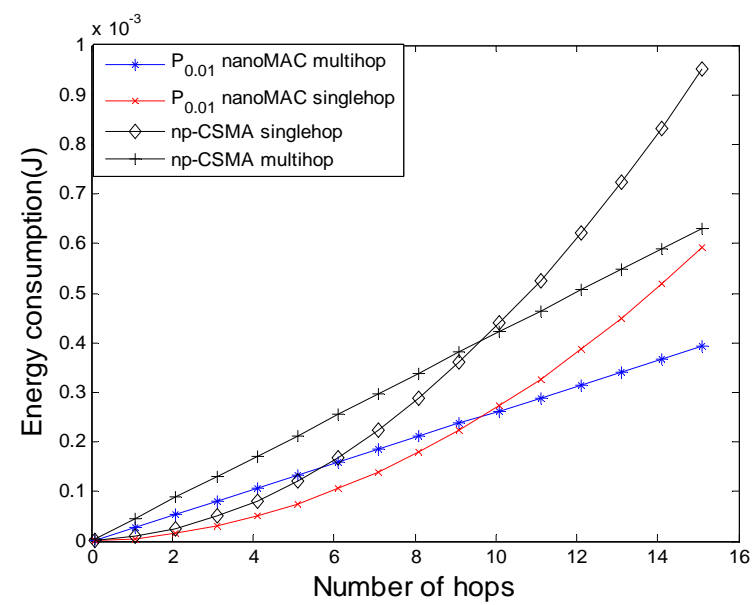

Fig.14. Energy consumption of single hop and multihop in inter-cluster domain

Thus the hybrid MAC protocol suggested can significantly reduce energy consumption in the sensor network. BMA MAC protocol when used for intra-cluster domain and nanoMAC protocol when used for inter-cluster domain can achieve high energy savings when compared to conventional TDMA, ETDMA and np-CSMA schemes.

\section{CONCLUSION}

The performance of the novel hybrid MAC protocol in terms of energy and delay with offered traffic load has been explored for the cluster based wireless sensor network. From the simulation results it is evident that for the intra-cluster communication, BMA protocol performs best and achieves 25\% reduction in energy consumption compared to TDMA and 5\% reduction in energy consumption than E-TDMA scheme and provides $15 \%$ less packet transmission delay by incorporating proper dynamic scheduling schemes. NanoMAC protocol provides better performance for inter-cluster communication and its energy expended for data transmission is almost $40 \%$ less than np-CSMA protocol. The delay of nanoMAC protocol is considerably reduced by $15 \%$ without any degradation in throughput when compared with np-CSMA scheme. This reduction in energy consumption and delay of the hybrid MAC protocol can significantly prolong the lifetime of the sensor network. 


\section{REFERENCES}

[1] L.Akyildiz, W.Su, Y.Sankarasubramanian and E.Cayirci, "A survey on sensor networks", IEEE Communications Magazine, vol.40, no.8, pp.102-114, 2002.

[2] I.Demirkol, C.Ersoy and F.Alagoz, "MAC protocols for wireless sensor networks", IEEE Communications Magazine, vol.44, no.4, pp.115-121, 2006.

[3] Leonard Kleinrock and Found A.Tobagi, "Packet switching in radio channels: Part I - Carrier sense multiple-access modes and their throughput-delay characteristics", IEEE Transactions on Communications, vol.23, no.12, pp.1400-1416, 1975.

[4] A.Hoiydi, "Spatial TDMA and CSMA with preamble sampling for low power ad hoc wireless sensor networks", Proceedings of the 7th International Symposium on Computers and Communication, Italy, pp.685-692, 2002.

[5] Wendi.B.Heinzelman, Anandha.P.Chandrakasan and Hari Balakrishnan, "An application -specific protocol architecture for wireless micro sensor networks", IEEE Transactions on Wireless Communications, vol.1, no.8, pp.660-670, 2002.

[6] I.Rhee, A.Warrier, M.Aia and J.Min, "Z-MAC: a hybrid MAC for wireless sensor networks", IEEE Transactions on Networking, vol. 16, no.8, pp.511-524, 2008.

[7] J.Li and G.Y.Lazarou, “ A bit-map assisted energy-efficient MAC scheme for wireless sensor networks", Proceedings of the 3rd IEEE International Symposium on Information Processing in Sensor Networks, New York, pp.55-60, 2004.

[8] J.Ansari, J.Riihijarvi, P.Mahonen and J.Haapola, "Implementation and performance evaluation of nanoMAC: a low-power MAC solution for high density wireless sensor networks", International Journal of Sensors, vol.2, no.5 pp.341-349, 2007

[9] V.Bacci, F.Chiti, S.Morosi, J.Happola and Z.Shelby, "Performance evaluation of optimized medium access control schemes based on ultra wideband technology", Proceedings of the 17th IEEE International Symposium on Personal, Indoor and Mobile Radio Communications, Finland, pp.1-6, 2006.

[10] J.Haapola, "MAC energy performance in duty cycle constrained sensor network and effect of sleep", Proceedings of IEEE International Workshop on Wireless Ad-Hoc Networks, Finland, pp.320-324, 2004.

[11] Z.Shelby, C.Raez and J.Happola, "Energy optimization in multihop wireless embedded and sensor networks", Proceedings of 15th IEEE
International Symposium on Personal, Indoor and Mobile Radio Communications, Spain, pp.221-225, 2004

J. Vidhya received B.E degree in Electronics and Communication Engineering from Bharathidasan University, Trichy in 1999 and M.Tech

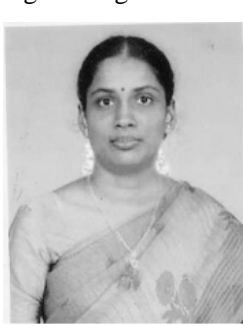
degree in Electronics and Communication Engineering from Pondicherry University in 2001. She is pursuing her Ph.D. programme in Department of Electronics and Communication Engineering, Pondicherry University. She is currently working as Assistant Professor in Department of Electronics and Communication Engineering at Rajiv Gandhi College of Engineering and Technology, affliated to Pondicherry University. Her Wireless ad hoc and sensor networks.

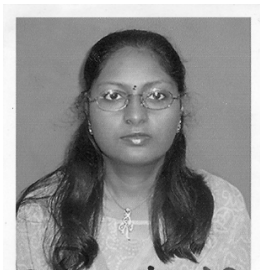

G. Kalpana received B.Tech degree in Electronics and Communication Engineering from Pondicherry University in 2006. She is pursuing her M.Tech in Pondicherry Engineering College, Pondicherry. Her area of interest includes Wireless data communication and Computer networks.

P. Dananjayan received Bachelor of Science from University of Madras in 1979, Bachelor of Technology in 1982 and Master of Engineering in 1984 from Madras Institute of Technology, Chennai and Ph.D. degree from Anna University, Chennai in 1998 $\mathrm{He}$ is working as Professor and Head of the Department of Electronics and Communication Engineering, Pondicherry Engineering College, Pondicherry, India. $\mathrm{He}$ has more than 60 publications in National and International Journals. He has presented more than 130 papers in National and International conferences. He has guided $6 \mathrm{Ph} . \mathrm{D}$ candidates and currently guiding eight $\mathrm{Ph} . \mathrm{D}$ students. His research interests include Spread spectrum techniques, Wireless communication and Sensor networks. 